IVb. NEUTRON STELLAR EVOLUTION

Evolution in Binaries

CHAIR: A. BZaauw 


\title{
BINARY PULSARS: OBSERVATIONS AND IMPLICATIONS
}

\author{
J. H. Taylor \\ Joseph Henry Laboratories and Physics Department \\ Princeton University \\ Princeton, NJ 08544 USA
}

\begin{abstract}
The Galaxy contains a large number of neutron stars in gravitationally bound binary systems. Among the most fruitful of these to study have been the binary radio pulsars, of which seven are now known. Unlike the "accretion-powered" neutron stars located in mass-exchanging $\mathrm{X}$-ray binary systems, the "rotationpowered" binary radio pulsars are found in dynamically simple, clean systems in which both stellar components have already completed their nuclear evolution, thereby shedding their atmospheres and most of their mass. In such circumstances the orbital parameters of the system and the rotational parameters of the pulsar can be determined with high precision from analysis of pulse timing data. These measurements constrain the component masses and yield an estimate of the pulsar's magnetic dipole moment, which turns out to be an essential parameter in understanding the evolution of the systems. In this paper I review the known facts concerning binary pulsars, and then briefly discuss some implications for our understanding of the place of neutron stars in stellar evolution.
\end{abstract}

Let me first describe the distribution of binary pulsar systems in the Galaxy. The galactic coordinates of all 430 presently known pulsars are illustrated in Figure 1, with a rough indication of dispersion measure (and hence relative distance) encoded in the sizes of the circles. Locations of the seven binary pulsars and the millisecond pulsar PSR $1937+21$, which I believe is closely related to the binaries, are explicitly identified. Five of the eight named objects lie at very small galactic latitudes $|b|<$ $4^{\circ}$, and the remaining three have small dispersion measures and thus relatively small distances from the Sun. Based on distance estimates determined from the model of Lyne, Manchester \& Taylor (1985), the mean distance of the group from the galactic plane is $\langle|z|\rangle=165 \mathrm{pc}$, about half the corresponding scale height of the pulsar population as a whole. Their smaller $z$-distances imply that pulsars in binary systems have smaller peculiar velocities than single pulsars, a conclusion sure to be of significance in understanding their evolution. 


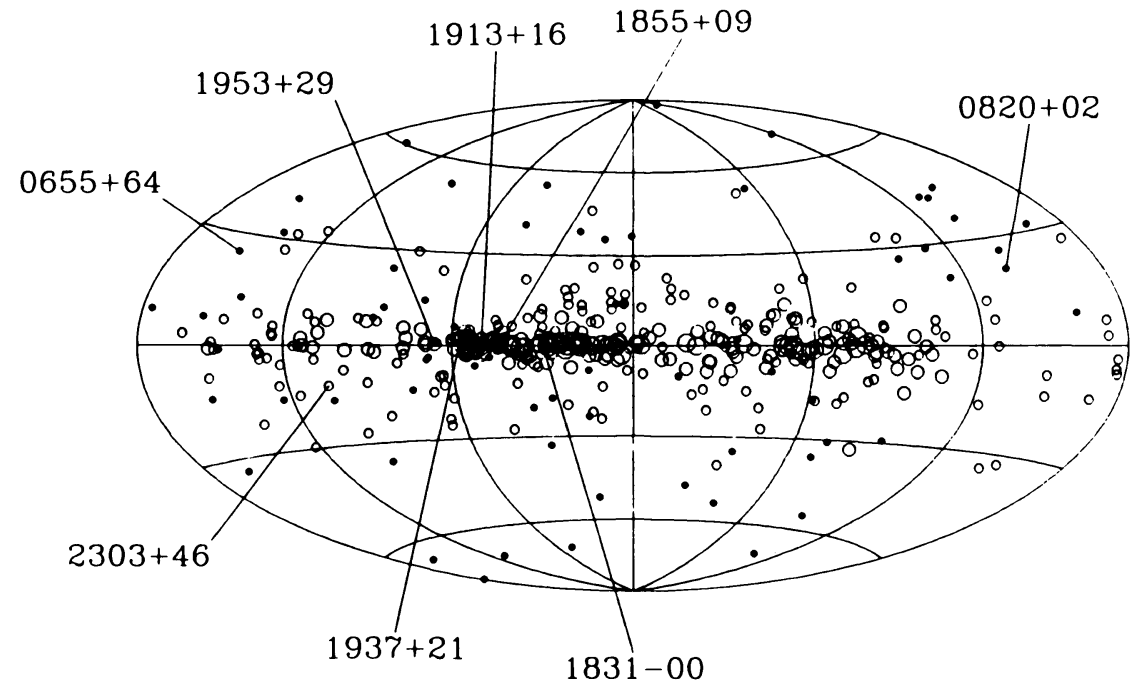

Fig. 1. Distribution of 430 pulsars in galactic coordinates. Sizes of circles correspond to dispersion measures in the ranges $<30,30$ to 100,100 to 300 , and $>300 \mathrm{~cm}^{-3}$ pc. Binary and millisecond pulsars are identified by name.
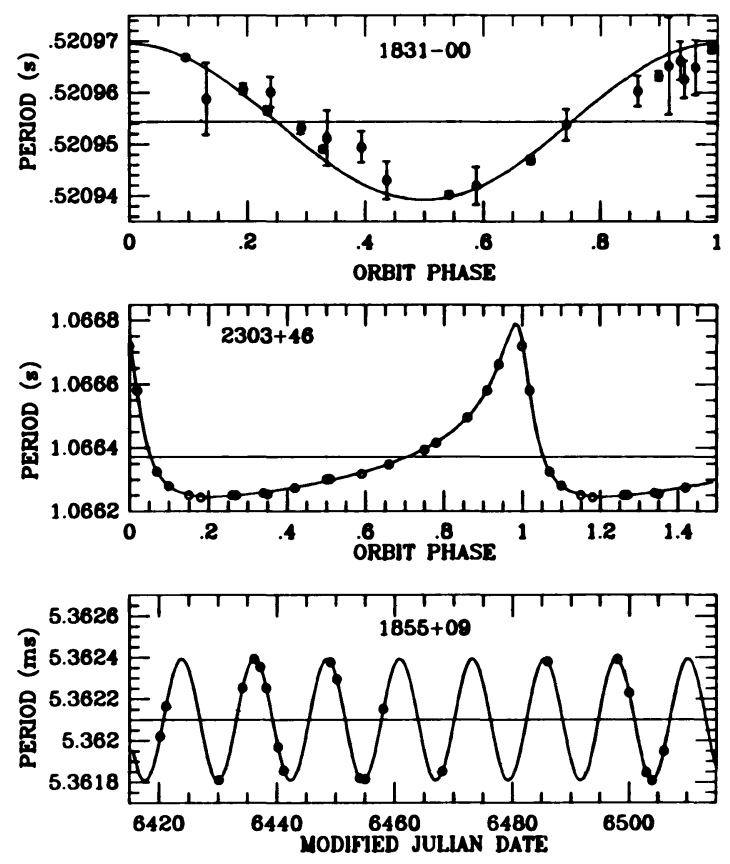

Fig. 2. Velocity curves of the three most recently discovered binary pulsars (Dewey et al. 1986, Stokes et al. 1985, Segelstein et al. 1986). 
Table I. Parameters of binary and millisecond pulsars.

\begin{tabular}{|c|c|c|c|c|c|c|c|c|c|}
\hline PSR & $\begin{array}{c}P \\
(\mathrm{~ms})\end{array}$ & $\log \dot{P}$ & $\begin{array}{r}\log B \\
(\mathrm{G})\end{array}$ & $\begin{array}{l}|z| \\
(\mathrm{pc})\end{array}$ & $\begin{array}{c}a \\
\left(\mathbf{R}_{\odot}\right)\end{array}$ & $\begin{array}{c}P_{b} \\
\text { (days) }\end{array}$ & $e$ & $\begin{array}{c}f\left(m_{1}, m_{2}\right) \\
\left(\mathbf{M}_{\odot}\right)\end{array}$ & $\begin{array}{c}\text { Likely } m_{2} \\
\qquad\left(\mathbf{M}_{\odot}\right)\end{array}$ \\
\hline $1937+21$ & 1.6 & -19.0 & 8.6 & 20 & - & - & - & - & - \\
\hline $1855+09$ & 5.4 & -19.7 & 8.5 & 20 & 23 & 12.33 & 0.00002 & 0.0052 & $0.2-0.4$ \\
\hline $1953+29$ & 6.1 & -19.5 & 8.6 & 20 & 100 & 117.35 & 0.0003 & 0.0027 & $0.2-0.4$ \\
\hline $0655+64$ & 195.6 & -18.2 & 10.0 & 120 & 5 & 1.03 & $<0.00005$ & 0.0712 & $0.7-0.8$ \\
\hline $1913+16$ & 59.0 & -17.1 & 10.3 & 190 & 2.8 & 0.32 & 0.6171 & 0.1322 & 1.38 \\
\hline $1831-00$ & 520.9 & $<-17.0$ & $<10.9$ & 190 & 6 & 1.81 & $<0.005$ & 0.00012 & $0.06-0.13$ \\
\hline $0820+02$ & 864.9 & -16.0 & 11.0 & 280 & 500 & 1232.40 & 0.0119 & 0.0030 & $0.2-0.4$ \\
\hline $2303+46$ & 1066.4 & -15.4 & 11.8 & 480 & 29 & 12.34 & 0.6584 & 0.2463 & $1.2-1.8$ \\
\hline
\end{tabular}

Timing data for binary pulsars yield the same kind of Doppler information as that available for single-line spectroscopic binary stars. Three examples of binary pulsar velocity curves are shown in Figure 2. In practice, analysis of the orbits is carried out using absolute pulse arrival times rather than inferred velocities, since the available precision is much higher with this approach (Taylor et al. 1976). Good orbital solutions are now available for all seven binary pulsars, and Table I summarizes some of the astrophysically interesting parameters.

As can be seen in the table, orbital periods of the seven binary pulsars range from less than eight hours to more than three years. Five of the orbits are essentially circular, with eccentricities $e \lessgtr 0.01$. Four of these also have very small mass functions

$$
f_{1}\left(m_{1}, m_{2}\right)=\frac{\left(m_{2} \sin i\right)^{3}}{\left(m_{1}+m_{2}\right)^{2}},
$$

implying companion star masses no more than a few tenths of a solar mass. (Here $m_{1}$ and $m_{2}$ are the masses of the pulsar and companion, respectively, and $i$ is the inclination between the plane of the orbit and the plane of the sky.) The remaining two systems, in contrast, have orbits with $e>0.6$ and much larger mass functions. Table I also lists the approximate separations between the pulsars and their companion stars, computed (except for PSR 1913+16) under the assumptions $m_{1}=1.4 M_{\odot}$ and $\cos i=0.5$. The estimated separations range from 2.8 to 500 solar radii.

Masses of the component stars are of particular importance, but unfortunately are not uniquely determined by the mass function. However, Equation (1) provides a useful constraining relation between $m_{1}, m_{2}$, and $i$, as illustrated for each of the seven systems in Figures 3 through 5. Figure 3 shows curves of $m_{2}$ vs. $m_{1}$ 
for assumed values of $\cos i=0.0,0.5$, and 0.8 . With no other information on $i$ available, one can only conclude that there is a $50 \%$ chance that the correct masses lie between the lower two curves in each sub-figure, and an $80 \%$ chance that they lie between the top and bottom curves. Under the reasonable assumption that the pulsar mass is close to the Chandrasekhar mass, $1.4 M_{\odot}$, it is clear that the companions of PSRs 0820+02, 1831-00, 1855+09, and 1953+29 are all low-mass objects, probably in the range $\sim 0.06$ to $0.4 M_{\odot}$.

For the remaining three binary pulsars some additional information on the masses is available. Lyne (1984) has used interstellar scintillation observations to estimate the transverse velocity of PSR $0655+64$ as a function of orbital phase. Combined with timing results for the radial velocity, his data yield two possible solutions for the orbital inclination. These values constrain the component masses as illustrated in Figure 4. For a pulsar mass of $1.4 M_{\odot}$, the companion of PSR $0655+64$ must have a mass very close to either $0.66 M_{\odot}$ or $0.78 M_{\odot}$.

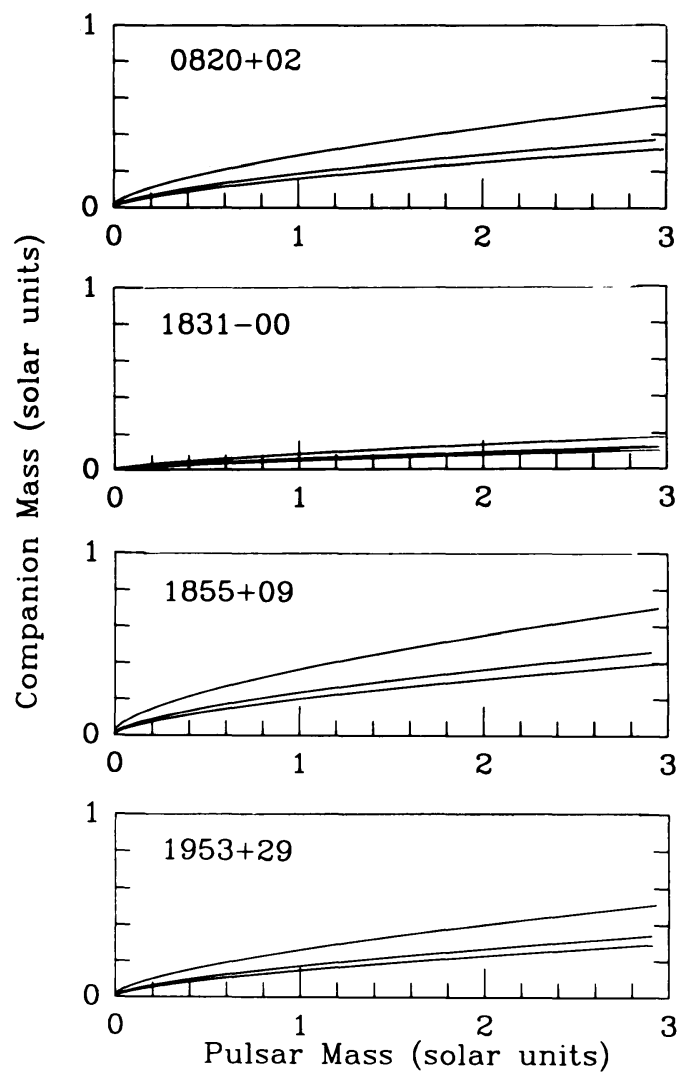

Fig. 3. Constraints on the masses of PSRs 0820+02, 1831-00, 1855+09, and $1953+29$ and their companion stars. In each panel the three curves correspond to assumed values $\cos i=0.0,0.5$, and 0.8 (from bottom to top). 


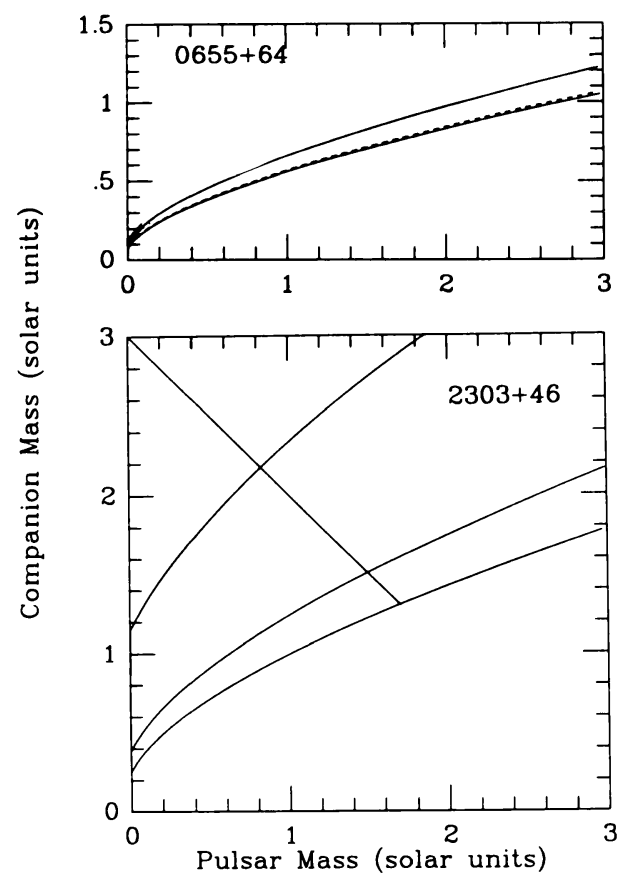

Fig. 4. Constraints on the masses of PSRs $0655+64$ and $2303+46$ and their companion stars. For $0655+64$ the masses must lie on or close to one of the two heavy curves, which correspond to the values $\cos i=0.10$ and $\cos i=0.47$ consistent with the scintillation measurements of Lyne (1984). The lighter curves indicate ranges of uncertainty. For $2303+46$, as in Fig. 3, the three curves correspond to assumed values $\cos i=0.0,0.5$, and 0.8 . The observed rate of periastron advance constrains the masses of this system to lie on or neâr the sloping straight line (see text).

My colleagues and I have recently succeeded in measuring the general relativistic periastron advance of the orbit of PSR $2303+46$, one of the two binary pulsars with high orbital eccentricity and large mass function. For this system, as for PSR $1913+16$, there can be little doubt that it consists of two neutron stars. The rate of periastron advance, currently estimated as $\dot{\omega}=0.0109 \pm 0.0023$ (Taylor et al. 1986), implies a total system mass $m_{1}+m_{2}=3.0 \pm 0.9 M_{\odot}$ - a very plausible result for the sum of two neutron stars. The sloping straight line in Figure 4 illustrates for PSR $2303+46$ the additional constraint imposed by $m_{1}+m_{2}=3.0 M_{\odot}$. Much better precision should be obtainable for $\dot{\omega}$ within a year or so.

PSR 1913+16, the best studied of all binary pulsars, has the ideal combination of short orbital period, short pulsar period, and large eccentricity which combine to make possible the detection of higher-order secular and periodic relativistic effects, 
and therefore to specify the component masses quite accurately. In addition to the rate of precession of the orbit, it is possible to determine the variable part of secondorder Doppler shift and gravitational redshift, $\gamma$; the rate of decay of the orbit due to gravitational radiation, $\dot{P}_{b}$; and the orbital inclination, $\sin i$. The most recent analysis (Taylor 1986, Taylor \& Weisberg 1986; see also Weisberg \& Taylor 1984) yields for the masses $m_{1}=1.451 \pm 0.007$ and $m_{2}=1.378 \pm 0.007$ (see Figure 5). These results are extremely important in that they provide a firm experimental foundation on which to build models of neutron stars and their formation.

$1913+16$

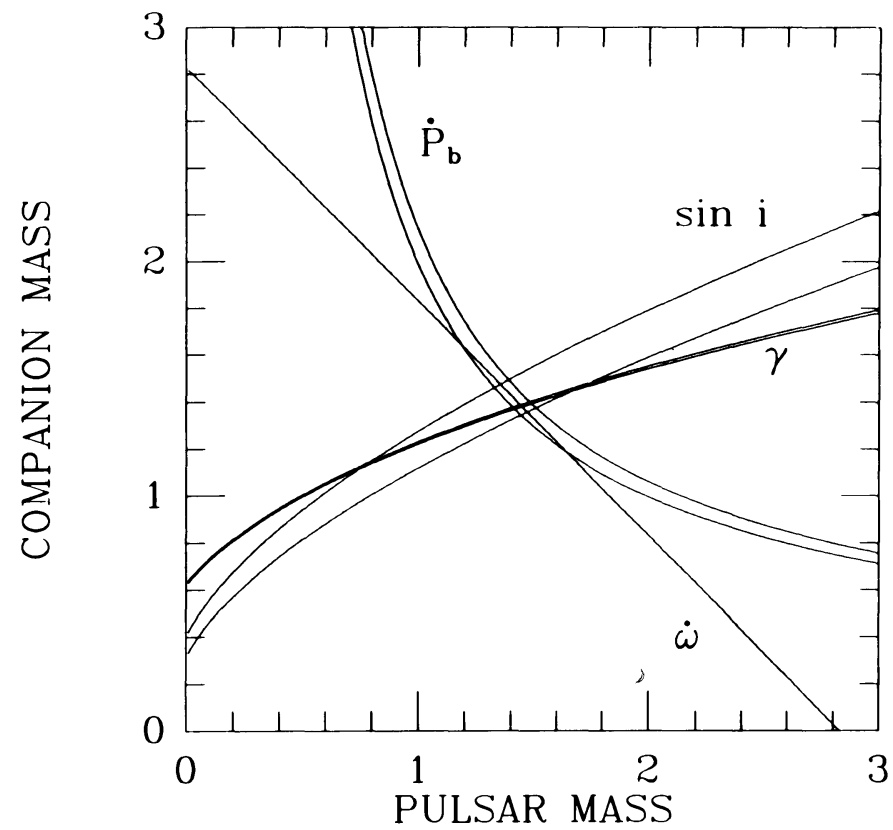

Fig. 5. Constraints on the masses of PSR $1913+16$ and its companion. Families of curves bracket the range of masses consistent with each of the relativistic parameters $\dot{\omega}, \gamma, \dot{P}_{b}$, and $\sin i$. (The uncertainties of $\dot{\omega}$ and $\gamma$ are too small to show.)

Let me now turn attention from orbital and dynamical matters to the characteristics of the binary pulsars themselves. Figure 6, an up-to-date version of the pulsar $P, \dot{P}$ diagram, shows that pulsars found in binary systems have unusually small spin-down rates $\dot{P}$, and that most of them have unusually small periods $P$ as well. There is some evidence that their luminosities are somewhat smaller (by a factor of about three) than those of single pulsars, though the large dispersion of pulsar luminosities makes it difficult to state this with confidence. A better statement concerning luminosities may be that pulsars located close to the "death line" 
in Figure 6 (Ruderman \& Sutherland 1975) tend to have smaller radio luminosities (Taylor \& Stinebring 1986; see also Prószyński \& Przybycień 1984). All of the binary pulsars either have unusually short periods or are found not far from the death line.

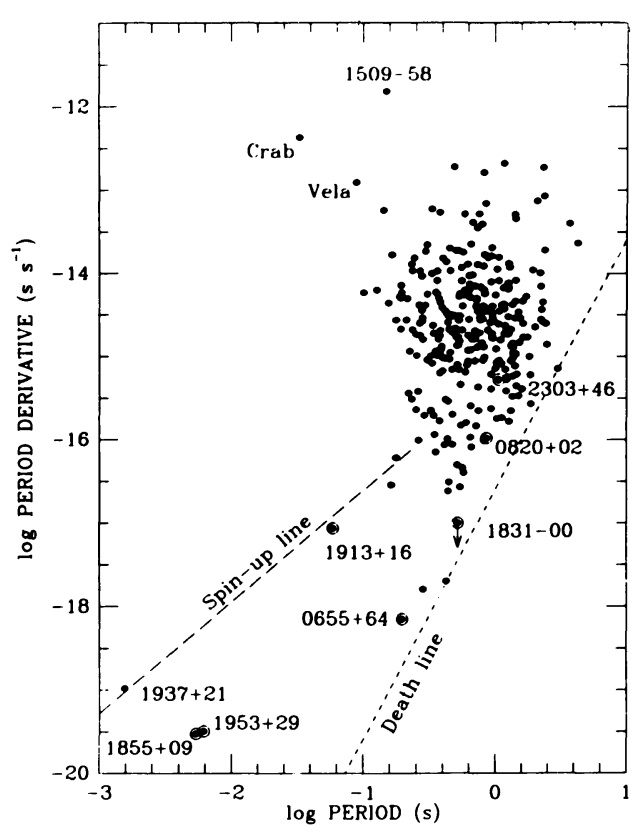

Fig. 6. Periods and period derivatives of 349 pulsars.

Pulsars with small values of $\dot{P}$ generally show little if any timing noise (Cordes and Downs 1985). Although in some cases the span of observations is not yet very long, no evidence for any timing noise has yet turned up in our timing observations of the binary and millisecond pulsars. PSRs $1855+09$ and $1937+21$, in particular, appear to be extraordinarily stable clocks (Davis et al. 1985, Segelstein et al. 1986). This fact is consistent with the understanding that as recycled objects, binary pulsars are likely to be very old. It is probable that most, if not all of them exist in the "active" region of Figure 6 (to the left of the death-line) solely because they acquired extra angular momentum while accreting matter from their evolving companions.

Other characteristics of the binary radio pulsars appear to be indistinguishable from those of single pulsars. Pulse shapes and pulse widths are normal; average polarization characteristics, when they have been measured (e.g. Stinebring et al. 1984, Segelstein et al. 1986), appear to be unextraordinary. 
Although fewer than $2 \%$ of the 430 presently known pulsars are in binary systems, the fraction increases to $13 \%$ (4 of 30) in the period range $P<200 \mathrm{~ms}$, and to $60 \%$ ( 3 of 5 ) of those with $P<60 \mathrm{~ms}$. Six percent of the 50 pulsars with the smallest dispersion measures, and therefore presumably closest to the sun, are binaries. Until very recently all major pulsar surveys were relatively insensitive for periods $\lesssim 200 \mathrm{~ms}$ (Dewey et al. 1984), and only one high-sensitivity survey has been made looking explicitly for low-luminosity pulsars in the solar vicinity (Dewey et al. 1985). It therefore seems likely that as more extensive surveys for fast and nearby pulsars are undertaken, many more binaries will be found. Experience gained in the Princeton/Arecibo survey (Segelstein et al. 1986; Stokes et al. 1986) suggests that at least $\sim 10 \%$ of the pulsars detected in a survey with good sensitivity down to $P \lessgtr 5 \mathrm{~ms}$ will be millisecond pulsars, and probably most of these will be binaries. Future fulfillment of these expectations will be a great boon for understanding of the physics of neutron stars and their evolution, and perhaps for gravitation physics as well.

\section{REFERENCES}

Cordes, J.M., and Downs, G.S. 1985, Astrophys. J. Supp. Ser., 59, 343.

Davis, M.M., Taylor, J.H., Weisberg, J.M., and Backer, D.C. 1985, Nature, 315, 547.

Dewey, R.J., Stokes, G.H., Segelstein, D.J., Taylor, J.H., and Weisberg, J.M. 1984, in Millisecond Pulsars, ed. S.P. Reynolds and D.R. Stinebring, (National Radio Astronomy Observatory: Green Bank, WV), p. 234.

Dewey, R.J., Taylor, J.H., Weisberg, J.M., and Stokes, G.H. 1985, Ap. J. (Letters), 294, L25.

Dewey, R.J., Maguire, C.M., Rawley, L.A., Stokes, G.H., and Taylor, J.H. 1986, Nature, in press.

Lyne, A.G. 1984, Nature, 310, 310.

Lyne, A.G., Manchester, R.N., and Taylor, J.H. 1985, M.N.R.A.S., 213, 613.

Prószyński, M., and Przybycień, D. 1984, in Millisecond Pulsars, ed. S.P. Reynolds and D.R. Stinebring, (National Radio Astronomy Observatory: Green Bank, WV), p. 151.

Rawley, L.A., Taylor, J.H., and Davis, M.M. 1986, Nature, 319, 383.

Ruderman, M.A., and Sutherland, P.G. 1975, Astrophys. J., 196, 51.

Segelstein, D.J., Rawley, L.A., Stinebring, D.R., Fruchter, A.S., and Taylor, J.H. 1986, Nature, in press. 
Stinebring, D.R., Boriakoff, V., Cordes, J.M., Deich, W., and Wolszczan, A. 1984, in Millisecond Pulsars, ed. S.P. Reynolds and D.R. Stinebring, (National Radio Astronomy Observatory: Green Bank, WV), p. 32.

Stokes, G.H., Segelstein, D.J., Taylor, J.H., and Dewey, R.J. 1986, Astrophys. J., in press.

Stokes, G.H., Taylor, J.H., Weisberg, J.M., and Dewey, R.J. 1985, Nature, 317, 787.

Taylor, J.H. 1986, 11th Intl. Conf. on General Relativity and Gravitation, Stockholm, July 1986.

Taylor, J.H., Dewey, R.J., Weisberg, J.M., Maguire, C.M. 1986, unpublished results. Taylor, J.H., Hulse, R.A., Fowler, L.A., Gullahorn, G.E., and Rankin, J.M. 1976, Astrophys. J. (Letters), 206, L53.

Taylor, J.H., and Stinebring, D.R. 1986, Ann. Rev. Astron. Astrophys., in press.

Taylor, J.H., and Weisberg, J.M. 1986, in preparation.

Weisberg, J.M., and Taylor, J.H. 1984, Phys. Rev. Letters, 52, 1348.

\section{DISCUSSION}

J. Arons: On Monday you remarked that you thought the millisecond pulsars are a different population. Could you elaborate, since they are part of the binaries?

J. Taylor: I believe that a bi-modal period distribution probably exists because most spin-ups will occur after a time long enough for the magnetic fields to have decayed to less than $10^{9}$ gauss, so the equilibrium periods will be very short.

J. Arons: Then how does $1913 \mathrm{fit}$ in, since it is an example of a pulsar in a binary with a period between 10 and $100 \mathrm{msec}$, exactly where you are suggesting there will be a gap?

J. Taylor: Pulsars like $1913+16$ are the cases in which the original mass ratio was close to unity, so much less time elapsed between the times when the two stars evolved off the main sequence.

J.H. Huang: You put two theoretical lines on $\log \mathrm{P}$ vs. $\log \dot{\mathrm{P}} \mathrm{p} \operatorname{lot}$; i.e., a death line for isolated pulsars and a spin-up line for binaries. Does it mean you need a special evolution from isolated pulsars to binaries when you put it together?

J. Taylor: No. The death line applies for any kind of pulsar, but pulsars which cross the death line while still in a binary system may get a second life after being spun up.

J. Dicke1: Any thoughts or comments yet on the implications of the fact that the two binaries in which both components are neutron stars have only 1 pulsar each? 
J. Taylor: There are many reasons why the companion neutron stars may not be observable as pulsars; probably the most likely are beaming effects and the rather short pulsar lifetime.

R. Narayan: If $2 \%$ of observed pulsars are in binary systems, and those are on the average harder to see, both because they are under-luminous and because many of them have short periods, then would you agree that a substantial fraction of the active pulsars in the Galaxy are in binaries? In fact, could one even say that a substantial fraction are millisecond binary pulsars?

J. Taylor: I think that $10 \%$ is a lower limit on this substantial fraction, and it could, indeed, be considerably higher. of course the lifetime of the millisecond pulsars is much longer so their birthrate could still be much smaller than that of ordinary pulsars.

L. Woltjer: You have 7 binaries with velocity amplitude larger than $8 \mathrm{~km} / \mathrm{sec}$. How many are single to the same level of accuracy?

J. Taylor: At least 340 of the known pulsars - essentially all of those with measured period derivatives, and which are not already known to be binaries - are certainly not binaries down to limits around one meter per second in velocity.

L. Woltjer: Do you have proper motions for any of the binaries?

J. Taylor: Only upper limits for proper motions are presently available for any of the binaries.

J. Shaham: Do you think that the $P P$ vs. $V_{t}$ relation for pulsars holds all the way down to the millisecond pulsars. If so, what does it imply regarding their expected abundance in the galaxy?

J. Taylor: Unless our ideas concerning the origin of millisecond pulsars are far wrong, they will not have large peculiar velocities. Within present uncertainties the abundance estimates will not be seriously affected; the z-distribution of millisecond pulsars would be made smaller by the low velocities, but larger by the long lifetimes. 